\title{
Anatomical Models: a Digital Revolution
}

\author{
John R. Fredieu ${ }^{1} \cdot$ Jennifer Kerbo $^{1} \cdot$ Mark Herron $^{2} \cdot$ Ryan Klatte $^{3} \cdot$ Malcolm Cooke $^{4}$
}

Published online: 28 March 2015

(C) The Author(s) 2015. This article is published with open access at Springerlink.com

\begin{abstract}
The use of three-dimensional (3D) anatomical models is ubiquitous in medical education. Medical educators rely on models to depict anatomical structures in a more efficient format than the cadaver; to move away from the clutter, discomfort, and complexity of a cadaveric dissection; and to clarify characteristics or functions of an anatomical structure that are not readily apparent in situ. Here, we review the use of physical anatomical models in teaching anatomical sciences in medical education. In addition, we examine the production of digital 3D models for interactive media and the production of physical models of anatomical structures using additive manufacturing (3D printing) methods. Finally, we examine methods of implementation of these visual and tactile resources in medical curricula. This review is intended as a primer for educators contemplating on the use of these learning objects in medical education.
\end{abstract}

Keywords 3D modeling · Additive manufacturing $\cdot 3 \mathrm{D}$ printing $\cdot$ Anatomy $\cdot$ Medical education

John R. Fredieu

jrf6@case.edu

1 Department of Anatomy, School of Medicine, Case Western Reserve University, 10900 Euclid Ave., Cleveland, OH 44106, USA

2 Case Western Reserve University School of Medicine, Cleveland, OH, USA

3 Lerner Research Institute, Cleveland Clinic, Cleveland, OH, USA

4 Department of Mechanical and Aerospace Engineering, Case Western Reserve University School of Engineering, Cleveland, OH, USA

\section{Introduction}

The use of three-dimensional (3D) anatomical models is ubiquitous in medical education. Medical educators rely on models to depict anatomical structures in a more efficient format than the cadaver [1-3] or when conforming to institutional constraints or social mores [4-6]. Anatomical models allow the user to move away from the clutter, discomfort, and complexity of a cadaveric dissection and can clarify characteristics or functions of an anatomical structure that are not readily apparent in situ. Models are very useful to explain anatomical relationships and function in structures that may be too small to discern adequately in a cadaver or that are constrained by other structures. Thus, anatomy education is enhanced and facilitated through the use of accurate anatomical models.

The use of anatomical models in medical curricula has been reported as effective in teaching and learning anatomy, although the form of the model and its presentation may impact efficacy in learning [3, 7-9]. Models can focus perspective on specific characteristics of an anatomical structure that are important in the educational objectives of the curriculum. For medical or dental students, accurate models are helpful in guiding cadaver dissection by providing an ideal view to assist in an approach to a structure or region. Anatomical models are important educational tools in institutions or settings that are unable to support the space, costs, or regulatory requirements required for cadaveric dissection or specimen storage. For these reasons, anatomy education will always benefit from a finely constructed 3D model.

The objective of the application of anatomical models in a curriculum is to either enable or enhance student learning. The model can be presented in a medical curriculum as a standalone learning asset or as part of a learning object, a collection of materials that help the student meet a specific learning objective $[10,11]$. However, studies have suggested that the 
impact of such materials varies by factors such as topic examined, visual and interactive modalities used, and student demographics and learning characteristics, and that individual computer-based educational materials must be assessed aggressively to ensure effective learning [12-15].

The anatomical model does not have to be physical. Digital $3 \mathrm{D}$ anatomical models have been reported to be effective in enhancing learning and retention in medical and dental students [16-20]. However, not all studies have supported this efficacy and the impact of digital 3D models on learning requires further examination [21, 22]. Similar to physical models, the impact on student learning of using digital 3D models in medical education is likely dependent on topic, presentation, and student learning styles.

In this review, we discuss the history of $3 \mathrm{D}$ models and the current state in the design and construction of digital and physical 3D models. In addition, we discuss the impact of these digital media and advances in additive manufacturing technology on the role of physical and digital 3D anatomical models in medical education. Finally, we discuss possible methods of implementing digital and printed 3D models in the medical school curriculum.

\section{History of the Anatomical Model}

The best "model" for investigating human anatomy has always been the human cadaver itself, because, in most cases, all the parts are there in the correct arrangement, the fine membranous and facial elements are intact, and the presentation of structures (soft, hard, smooth, rough, dry, moist) is accurate. It is safe to say that, from the beginning of curiosity, early man investigated wounds and organs of their dead brethren. However, in today's regulated and socially conscious institutions, access to a cadaver may be limited through budgetary or social issues, or, even if a cadaver is available, presentation of the desired cadaveric anatomy may be confusing, such as that of the pelvic spaces and fascia. Finally, body donation programs, storage, chemical and biological hazard compliance, and proper disposition of the cadaveric specimens may be daunting financial and logistical burdens for some institutions. These issues can be addressed through the use of fabricated anatomical models. Ancient and contemporary anatomical models range greatly in the detail and material (Fig. 1). They have advanced from simple wood or ivory representations of anatomical structures (Fig. 1a) to more detailed papier-mache or plaster models, to the intricate wax models of Susini, Towne, or Ziegler (Fig. 1b), and finally, to the present-day commercial plastic models (Fig. 1c) [1, 2, 6, 23-25]. The anatomical models also ranged in application including use by physicians to maintain discretion with female patients, training surgeons in lieu of cadavers, disrupting medico-political authorities, and of course, teaching anatomy $[4,5]$.

Physical anatomical models possess inherent limitations in their use, including costs, storage requirements, security, and maintenance. The costs of commercial models can reach thousands of dollars depending on material, size, detail, resolution, and interactivity. In addition, particularly with large student populations, physical anatomical models can be damaged, misplaced/stolen, or become just plain filthy from constant handling by students over decades. Finally, purchasing and maintaining the range of anatomical models required to depict all the variations and anomalies observed in human anatomy is not feasible. Thus, the use of physical anatomical models presents challenges in selectivity and cost.

With the development of plastination by Gunther von Hagens and the "edutainment" of anatomy [26-28], some faculty speculated as to the demise of fabricated models in preference for actual anatomical specimens that are rendered resistant to degradation and damage. Plastinized specimens have been shown to be effective teaching tools [29, 30]. However, legal/ethical liabilities in donation and waste, as well as costs of acquisition (can be thousands of dollars for regional anatomical specimens) and proper storage facilities impact their use in anatomy education [31]. In addition, most of the limitations in the use of physical anatomical models described above are also applicable to plastinized specimens.

\section{Digital 3D Models}

Speculation as to the demise of physical models has also been a response to the development of advanced digital 3D renderings of structures and specimens through medical imagery or digital 3D modeling. Digital 3D models of anatomical structures can be accessed on a computer, through mobile apps, or through stand-alone interactive workstations (i.e., Anatomage, Touch of Life). The availability of these materials in the gross anatomy laboratory and at study workstations may reduce the need for physical anatomical models or even printed atlases and may facilitate teaching by bringing the material to the dissecting table. However, costs and space requirements for a dedicated interactive 3D imaging system are significant ( $\$ 100,000$ for an Anatomage table and support media), and as always, the advanced technology is likely to become dated and unsupported over time. The use of iPads and mobile devices reduces the costs, but they depend on the development of apps that have been assessed as to their impact on learning.

An advantage of digital 3D models is that they can be manipulated temporally to depict changes in a structure or specimen with regard to age or developmental stage, the impact of disease or injury, or functional mechanics. Digital 3D models can depict morphogenesis of the heart or inner ear and 
Fig. 1 Anatomical models. a An interactive ivory model depicting internal organs. Likely German, but the date is unknown (photo courtesy of the Dittrick Medical History Center, Case Western Reserve University). b An example of a wax model of a midstage human embryo. These models were created in the studios of Friedrich Ziegler in the late 1800 s based on reconstructed embryos from the laboratory of Wilhelm His. c A commercial heart model (G13 Heart (C) 3B Scientific GmbH, Germany, 2014, www.3bscientific.com)
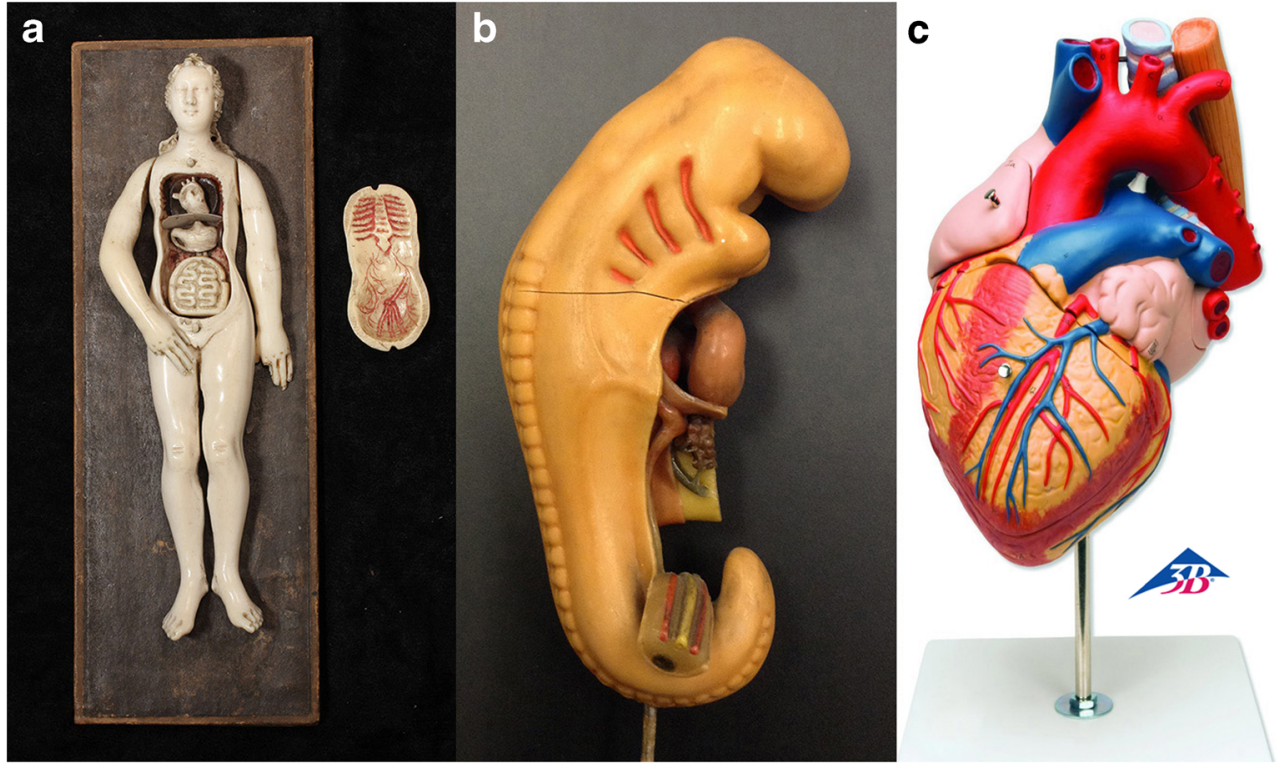

are able to clarify embryonic structures and tissues that are important during successive stages of embryogenesis. In addition, digital 3D models can track the course of liver cirrhosis or the progression of Alzheimer's disease from initial formation to stages of advanced tissue degradation. Finally, these models can demonstrate functional mechanics of joint movement in different positions or loads or the physiology of cardiac muscle function. In contrast, a series of physical models is required to achieve any of these demonstrations.

A clear advantage of digital 3D anatomical models is that any educator or institution can create them in a relatively short period of time, with the proper expertise (experienced biomedical illustrator). The costs can be relatively low, a workstation with free and open-source software. However, more effective software can be expensive ( $\$ 1000-\$ 5000 /$ year), even at academic pricing. Software purchasing or license fees depend on personal or institutional use, successful negotiation, and type of use (clinically approved or academic/educational). The creation and proliferation of model libraries or repositories may reduce the need for original models and drive the costs lower [32-34].

\section{Creation of Digital 3D Anatomical Models}

Digital 3D anatomical models can be created in a variety of ways including segmentation of clinical and research imaging, surface scanning, and 3D modeling, each of which has relative advantages and disadvantages.

\section{Creating a 3D Model Using Graphics Software}

3D modeling software can be used to construct a facsimile of an anatomical structure or region that can be manipulated with regard to orientation, size, resolution, color, movement, and time. The facsimile can be either schematic or realistic depending on the specific application of the model. For example, a model may depict laryngeal musculature and their function as levers and pulleys acting on a representative laryngeal skeleton, or as anatomically correct renditions of muscle and tendon with specific attachments to the laryngeal cartilages. These two types of models are very different in complexity, difficulty in design, and educational objectives.

An advantage of 3D modeling an anatomical structure is that specific characteristics of the structure can be enhanced or isolated such that the student can identify and focus on those structures. For example, a fabricated digital 3D model of a heart may enhance visualization of the conducting system, which cannot be segmented from CT or MRI data. However, in order to achieve this advantage in the control of the details of the 3D model, many revisions may be required, and of course, a talented biomedical illustrator is always a help.

Figure $2 \mathrm{a}, \mathrm{b}$ shows a heart model created using Maya (Autodesk). The exterior was sculpted from a box shape, while the interior structure was created separately then inserted into the exterior. Valve leaflets, chordae tendineae, and coronary arteries were created separately and joined to the interior or exterior. The model was constructed originally in a low polygon count for rough modeling, and then the polygon count was increased to incorporate more detail. This model can be used in multimedia presentations or further refined for creating physical models.

\section{Creating a 3D Model Using Imaging and Scanning}

Modeling software can use a series of photographs of an object to generate a 3D model. Autodesk 123D Catch, insight3d, and other open-source software can align images taken at 
Fig. 2 Surface (a) and mesh renders (b) of a heart model created using Maya and 3D Max (Autodesk, San Rafael, CA, USA)
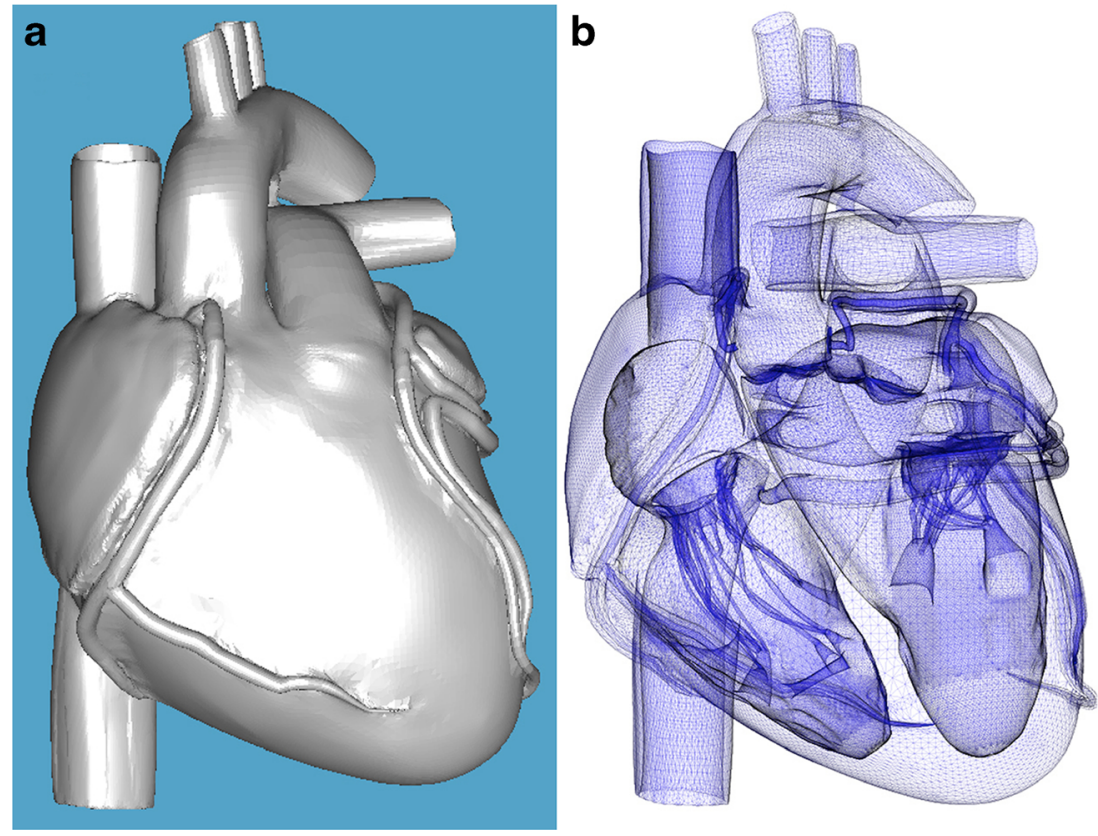

different angles from an object to create a digital 3D model of the object. The resolution and accuracy depend on the photographic parameters and the software capabilities and may be quite limited. Thus, a model of a liver or femur produced using this method may be more instructive than a model of an isolated sphenoid bone (Fig. 3a).

Digital 3D anatomical models may be created using laserbased 3D scanners or micro-CT, clinical CT, and MRI [35]. Laser-based 3D scanners range in price from a few hundred to many thousands of dollars and differ in resolution, specimen size, and ease of use. These devices can create sophisticated watertight 3D models that can be used as content in animations or in 3D printing. Laser-based scanners can be handheld, desktop, or industrial instruments that interpret the surface of an object either through laser triangulation, which uses reflected light from the surface of the object to calculate distance, or laser pulse-based/phase shift to measure time-offlight of reflected light to calculate distance. Models created from these methods depict surface structure only and may have resolution and accuracy concerns. Thus, the use of surface scanning in the creation of 3D models has limitations.

Micro-CT and clinical CT imaging use X-ray emissions, while MRI uses radio frequency-based emissions to generate cross-sectional images of an object that can be used to create $3 \mathrm{D}$ reconstructions of the object. Micro-CT imaging can produce precise digital models at a resolution of $0.5 \mu \mathrm{m}$ (Fig. 3b), while clinical CT and MRI can resolve structures on a submillimeter scale. In many cases, a clinical scan is sufficient to reconstruct anatomical structures such as organs and cavities [36] (Fig. 3c), but micro-CT may be required for detailed osteological analysis or the generation of high-resolution models. In both micro-CT and clinical CT, parameters may be adjusted to visualize internal structures of soft tissue and bone. These structures may be isolated through segmentation, as described below, to extract specific structures from within larger scans. Most digital 3D anatomical models are created from clinical CT imagery simply because patient data is collected using this modality and a large collection of these data exist. In addition, the chamber size of most micro-CT units is quite small and unable to scan long bones or large specimens.

MRI data can be used to generate digital 3D models of anatomical structures and readily detects soft tissue structure, which makes it more useful than $\mathrm{CT}$ in segmentation and extraction of soft structures. The advantages of MRI data are the detection of soft tissues, the large array of patient data that already exists, and the lack of exposure of the subject to radiation.

Disadvantages of these imaging methods are cost (a microCT scan of a sphenoid bone in an institutional core facility may cost \$200-300), scan size, and consent issues for patient data. Hospitals and clinics have large repositories of clinical CT and MRI patient data that, if organized properly, would offer a treasure trove of possible anatomical models for use in education. However, both Internal Review Board approval and close cooperation between radiologists, medical physicists, and educators are required. Public repositories of scanned material and segmented structures exist and are expanding (i.e., National Biomedical Imaging Archive (NBIA); Cornell Visualization and Image Analysis (VIA) group).

\section{Creating a 3D Model Using Segmentation}

While digital 3D models created by CT and MRI scanning can be quite detailed and can be modified to visualize specific 


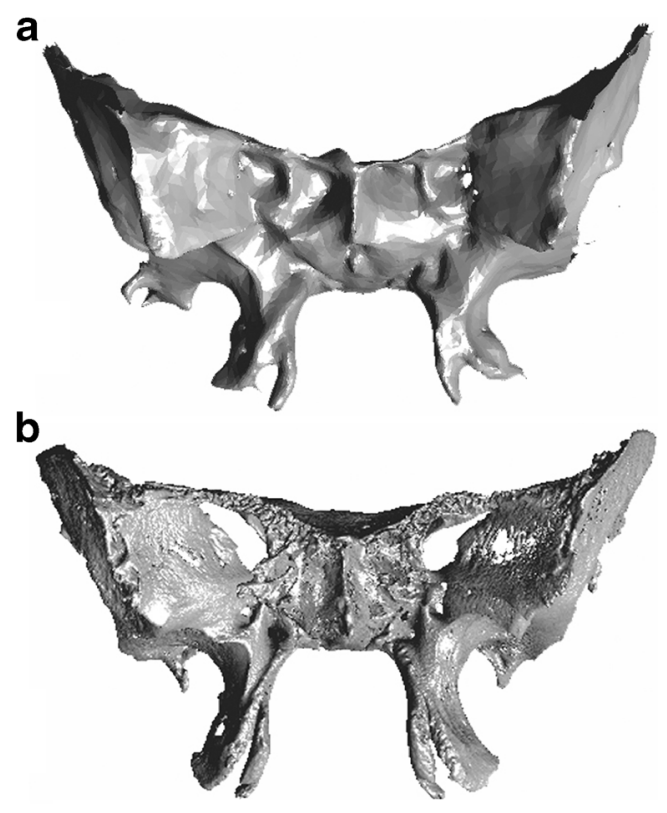

C

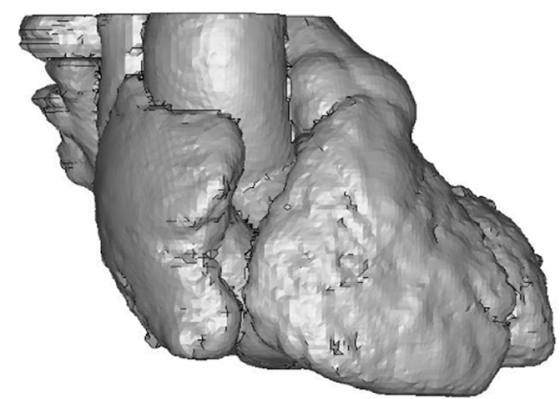

Fig. 3 Digital 3D models created using different methods. a Model of a sphenoid bone created using Autodesk 123D catch software (Autodesk, San Rafael, CA, USA). Resolution is poor and below that needed for use in education. b Model of a sphenoid bone obtained through micro-CT imaging and reconstruction using 3D Slicer (www.slicer.org). High resolution is obtained and the model can be used at size or scaled without loss of structure and resolution. c A 3D volume render of a heart from clinical CT imaging. Gross structure of the heart chambers is clear, while the epicardial surface and myocardial layers are missing

tissues, creating models of specific organs or tissues contained within the scan requires the use of segmentation. Segmentation assigns a label to pixels within an image that correspond to a specific structure. For example, the isolation and reconstruction of the hyoid bone in a head and neck CT or MRI scan series may be accomplished by the selection of pixels based on contrast differences between the bone and surrounding tissue in the appropriate sections. The selection of pixels can occur by either assigning a mask to select for pixels corresponding to the hyoid bone or tracing the hyoid bone field and excluding the rest of the data. Segmentation is relatively easy when isolating bone or the tracheal lumen because contrast thresholds can be a range that is sufficiently narrow to exclude unwanted pixels. However, soft tissues are more difficult to segment due to similar grayscale values on the image. For example, if the desired structure is the peritoneal membrane, contrast differences between the peritoneal membrane and the surrounding fascial and muscular layers may not be sufficient to automatically or semiautomatically segment into different structures. In this case, the structure must be manually segmented in each image, followed by digital reconstruction. Thus, substantial effort may be required to isolate and reconstruct some tissues.

There is an array of software (free ware and commercial) that enables segmentation and reconstruction of Digital Imaging and Communications in Medicine (DICOM) medical image stacks. The DICOM format is the international standard format for medical images (ISO 12052) and has replaced the use of X-ray film. Osirix, 3D-Slicer, and InVesalius are examples of free-ware or low-cost DICOM viewers that enable segmentation. A combination of Osirix free ware and the Virtual Human enables anyone to create 3D models of anatomical structures, but the resolution may be below that suitable for medical education. More powerful segmentation software solutions exist, such as Mimics (Materialise), but their costs can be substantial (can be $\$ 1-5000 /$ year depending on version, capabilities, and negotiation). The use of more powerful segmentation software cannot offset the issues of resolution of the image, but they can simplify the process through automation and creative segmentation [37-39].

The DICOM image reconstruction typically converts the segmented anatomy to a $3 \mathrm{D}$ mesh model, such as a stereolithography or standard tessellation language (STL) file. The STL file can be further processed into a nonuniform rational B-spline (NURBS) model as needed using appropriate software, including Geomagic (3D Systems) and Rhino (Robert McNeel \& Associates). Either the mesh or NURBS model can be edited in 3D modeling software to enhance features or correct problems encountered in segmentation. Mesh-based 3D modelers include Maya (Autodesk), Blender (The Blender Foundation), and Magics (Materialise); NURBS 3D modelers include Rhino and mainstream CAD software such as Autodesk Creative Suite (Autodesk) and Solidworks (Dassault Systèmes SolidWorks Corp.).

\section{Application of the Digital 3D Model}

Once the desired digital 3D anatomical model is obtained, it can be used in animations or as an object in an interactive format. Advantages that these models have over the cadaver or physical 3D models include their application in many educational formats (lectures, online material, and print) and portability (downloadable to any PC or mobile device). These models can be altered to enhance desired learning objectives or to conform to learner characteristics. These models offer a great advantage over static $2 \mathrm{D}$ images in terms of orientation and exploration of internal structure. For example, a 2D image of a heart may show extreme detail, but a student may be able to "fly through" a digital 3D model of the heart to obtain a 
clear view of the size and location of specific structures, as well as their relationship with surrounding anatomy. In addition, digital 3D models can be interactive or can depict a specific function (joint movement, ovulation).

\section{Using Digital 3D Anatomical Models to Create Physical Models}

A technological advance that may reduce the need for purchasing or maintaining a large library of physical 3D anatomical models is additive manufacturing. Additive manufacturing describes a field of fabrication technologies that build a part by the joining of materials layer by layer to produce a specific object [40]. Additive manufacturing technology (referred to here as 3D printing, a generic term) has advanced tremendously over the past two decades and has become functional in the development and construction of physical 3D anatomical models.

In general, 3D printers use the STL file format as the input for the build geometry. In order to successfully $3 \mathrm{D}$ print a model, the STL file needs to be watertight and free of other errors. For example, the model shown in Fig. 2a required preprocessing by propriety software that is vendor and printer specific. In this case, Insight (Stratasys), which is the preprocessing software used to analyze and prepare models for 3D printing on the Stratasys range of 3D printers, was used. Insight provides manipulation of a range of printer build parameters that determine the build quality and resolution of the finished model. This process was followed by a virtual build of the model at the individual layer level to assess the previously set parameters. This is typically an iterative process where some compromises are often made between speed, quality, and cost. For the model in Fig. 2a, this analysis initially indicated that some model features were too small to print appropriately. These errors were mainly a result of data conversion from the native 3D modeling format to a .stl representation, which is the required data format for the preprocessing software. Thus, an otherwise well-constructed model may require several adjustments in order to print using the desired method or material.

\section{Methods of 3D Printing}

Several different methods of 3D printing have been developed, and each has specific benefits and limitations in the creation of anatomical models (Table 1). Differences between the methods and the printers themselves include materials available, resolution, accuracy, repeatability, stability, costs, safety, size limitations, speed, and the number of materials per build. The importance of each of these parameters differs depending on the specific application of the printed object.

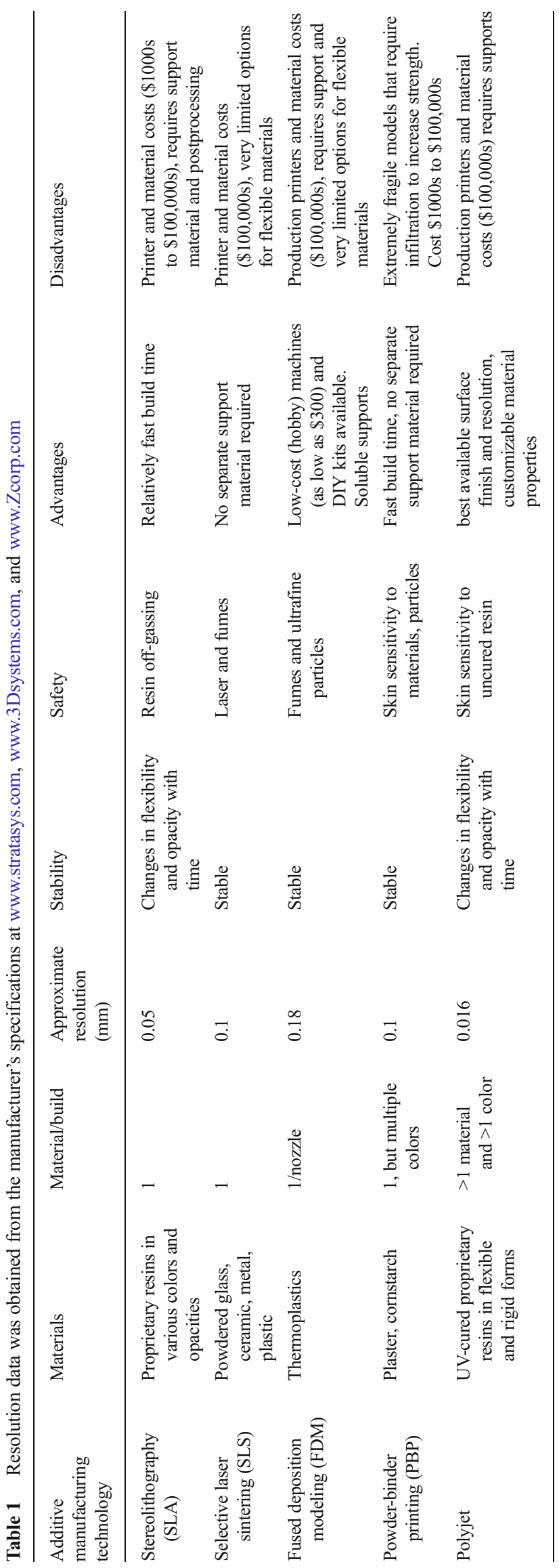


For the creation of anatomical models for education, resolution, stability, and cost are important parameters.

SLA uses an ultraviolet laser to cure photosensitive resin in sequential, thin, horizontally oriented layers that eventually construct the desired object [41]. Detailed models can be created in hours. However, the disadvantages of this method include costs of the printer ( $\$ 1000$ to $>\$ 100,000$, although prices are dropping), costs of the materials, postprocessing (removal of support material and curing), and long-term stability of the object.

Selective laser sintering (SLS) uses lasers to fuse powdered materials into a desired shape [42]. This method allows 3D printing in a variety of materials including powders of plastic, metal, glass, and ceramic. SLS does not require support materials since the unsintered material acts as support. Stability depends upon the material used.

Fused deposition modeling (FDM) uses thermoplastics extruded by a heated nozzle in a semiliquid state to produce layers of the desired object that then harden immediately after extrusion [43]. Different types of thermoplastic materials can be used that differ in hardness, flexibility, color, and translucency. Removable support material is required for smaller or protruding parts.

Powder-binder printers lay down a layer of powdered material followed by a binding agent. Colors can be applied selectively in the binder material. Distinct advantages are the printing speed and ability to print an object using multiple colors.

Polyjet technology builds 3D models by laying down photopolymer that is cured by UV. Speed and accuracy are advantages, but support material is required. In addition, the longterm stability of some materials can be a significant issue.

Although much improvement in costs, resolution, object scanning, image segmentation, and printing materials is needed for 3D printing to usurp the business model of commercial anatomical suppliers, these improvements may not be far away. The printer itself has been a costly investment, ranging from a few to hundreds of thousands of dollars. However, the cost is dropping rapidly with the development of desktop 3D printers $(<\$ 300)$ and more inexpensive materials (grass trimmer filament, recycled plastic bottles). The resolution of the printed object is also a consideration. Some higher end printers claim $<0.01 \mathrm{~mm}$ resolution, but the costs of such a printer can be a substantial burden to most institutions. The resolution of most desktop printers may be insufficient for printing a faithful replica of a structure as delicate as a sphenoid bone or an atrioventricular valve leaflet, especially if scaled to a smaller size. However, a nice articulated hand skeleton can be printed with attached musculature at these resolutions.

\section{Materials}

Many different materials can be used to construct a 3D printed object. In addition, several different materials or colors can be used. This ability allows the construction of complex models with hard, soft, opaque, and transparent components. Thermoplastics, including acrylonitrile butadiene styrene (ABS) and polylactic acid (PLA), ferrous and nonferrous metals, ceramic, elastic polymers, and many opaque, colored, and transparent proprietary materials can be used in the construction of an object, along with support materials that can be dissolved or washed away in postproduction processing. The choice of material must be made on esthetic, structural, and practical parameters. Strength, color, flexibility, opacity, stability, and costs must be considered. In addition, the printing process may require postprocessing of the object to ensure strength, durability, or transparency.

Examples of materials are shown in Fig. $4 \mathrm{a}, \mathrm{b}$. The heart model described above was printed on a Stratasys Fortus 400 in ABS with PLA as support material (Fig. 4a) and on a Stratasys Connex 350 in TangoPlus (Stratasys) with a separate removable material as support (Fig. 4b). Internal chambers, valve leaflets, chordae tendineae, and coronary artery lumen are demonstrated in both the ABS model and the flexible TangoPlus print (Fig. 4c, d). The TangoPlus model required printing in a split model in order to remove support material (nonpolymerized material) efficiently without damage to the fine structures. The removal of support material should be considered in model design, in particular in printing fine structures or using fragile materials.

\section{Resolution and Accuracy}

The resolution of a printed object is dependent upon the resolution of the digital 3D model, the resolution that can be achieved by the printer itself, and the material used in printing. Using a poorly constructed or low-resolution digital 3D model on an expensive, high-resolution printer will produce poorly constructed and low-resolution physical 3D models. Conversely, well-constructed 3D models may not be able to overcome the limitations of a low-resolution less expensive printer. Finally, the choice of material can affect resolution if the material cannot be extruded or cured in a sufficiently small amount or pattern or if the material cannot be supported properly during object construction.

The accuracy of a printed model in the representation of the digital model is dependent on the printing method, the capabilities of the specific printer, and the material used [44]. Accuracy with $\pm 0.0-1.0 \%$ seems to be the range of commercial printers; however, maintaining those tolerances requires calibration and maintenance. Different materials have a different impact on accuracy both at the time of printing and over time after printing. ABS, once cooled, is very stabile in configuration and appearance over time. However, polyjet and SLA resins may show alterations in dimension, opacity, and flexibility. In anatomical model construction for medical education, dimensional accuracy may not be as important as 
Fig. 4 Physical 3D models printed using FDM and polyjet methods and the digital model depicted in Fig. 2. a Complete model printed in ABS on a Stratasys Fortus 400 printer (Stratasys, Rehovot, Israel). b Internal structure of the model depicted in a (valve leaflets, chordae tendinae, and papillary muscles) is visible. c Complete model printed in TangoPlus on a Stratasys Connex 350 (Stratasys, Rehovot, Israel). d Internal structure of the model depicted in c (valve leaflets, chordae tendinae, and papillary muscles) is visible

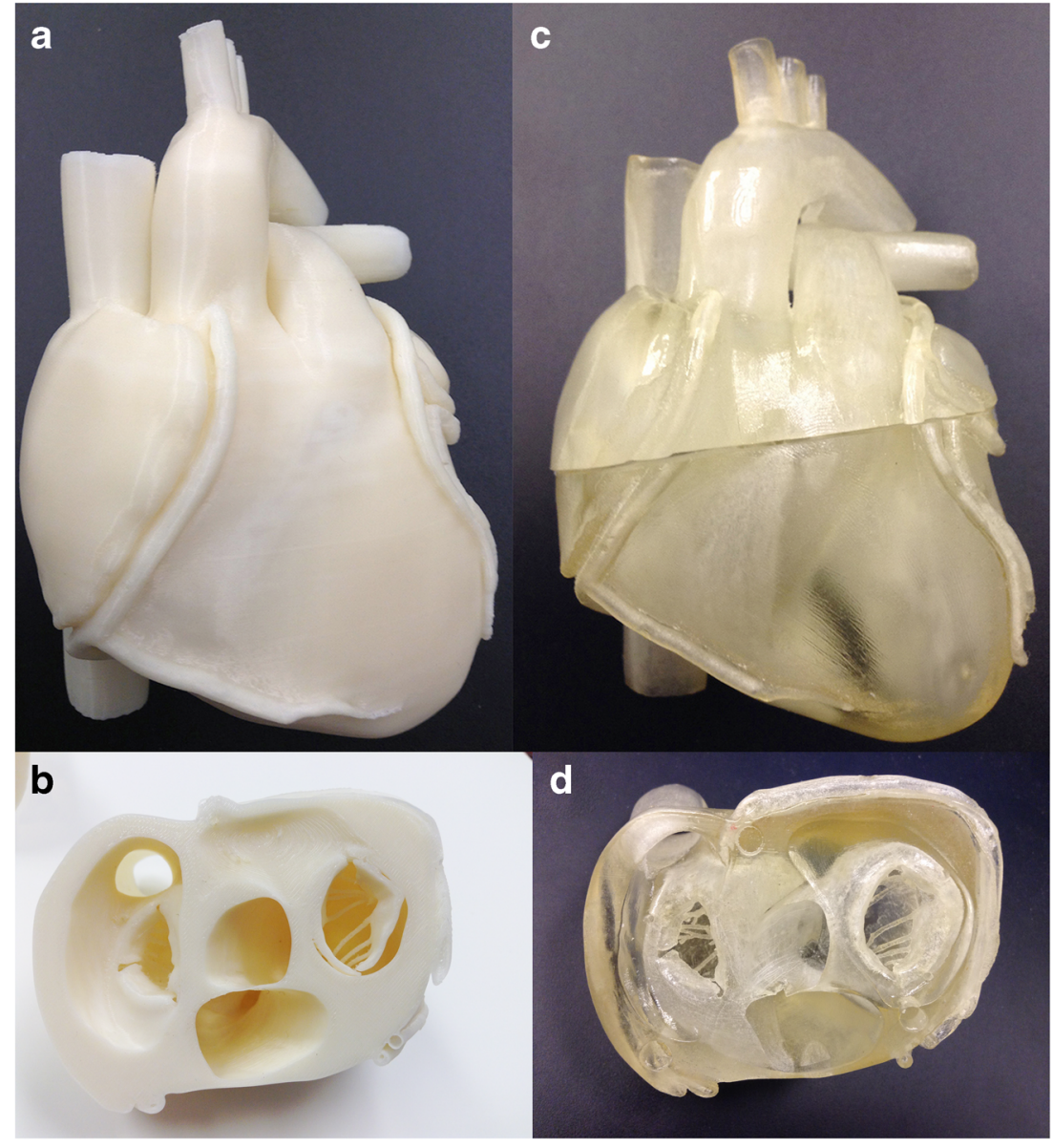

resolution and stability, since there is much variation in anatomical structure.

Desired resolution and accuracy as well as required stability affect the choice of printer and material. These parameters may also affect the speed of the printing process and the amount of materials used. These parameters also affect the cost of the printed object. Trade-offs between resolution and costs must be assessed individually to avoid a negative impact on accuracy or usefulness of the model.

\section{Costs}

The costs of a printed object are dependent upon the size and complexity of the 3D model, the choice of material used, and the cost to operate the printer. Commercial models offer some guarantees of quality and accuracy that can be assured through the inspection of the model prior to purchase. The creation of 3D models by faculty for use in local curricula may require several versions to achieve a suitable digital 3D model and printed 3D object. At this time, these costs may indicate that a commercial model is a better choice. Creating and printing a fabricated digital 3D heart model with accurate internal structure may cost thousands of dollars in artist and engineer time, print costs, and assessment of educational impact. Of course, many models can be printed after achieving a good design, which would decrease the costs overall. However, if $3 \mathrm{D}$ printing is to be a significant educational technology in medical and dental education, costs need to be reduced drastically.

\section{Safety Concerns}

There are safety concerns with the use of 3D printers. Some printers use high voltage and can have extremely hot surfaces, which presents obvious concerns. The results of a recent study suggest that some 3D printers emit ultrafine particles [45]. Particles of similar size have been shown to find their way into pulmonary airways, and even the brain, resulting in respiratory and neurological symptoms [46-49]. In addition to particles, heating some plastics, as in FDM, releases toxic compounds including benzene, carbon monoxide, hydrogen cyanide, and hydrogen chloride, which may be harmful in sufficient concentrations $[50,51]$. These are not trivial concerns since the detrimental health effects of ultrafine particle inhalation or toxic compound exposure may not become apparent for many years. Most commercial printers operate in a closed compartment or environment that can contain or filter the emitted particles or fumes. However, unless specific ventilation systems are in place, 3D printing should be performed 
cautiously. This is especially true when using open DIY printers. In addition, postprocessing may require caustic chemicals, sanding, or coating, which present safety hazards as well.

\section{Evidence of Efficacy in Learning}

A digital 3D model offers advantages over digital 2D or physical models in interactivity, perspective, access, and cost, either as a stand-alone learning asset or as part of a larger digital learning object. Studies have examined the use of digital learning objects in education [52,53], and several have even considered these materials in medical education $[10,13,18$, $19,22]$. Evidence has been presented for positive, negative, and neutral impacts of digital models on student learning [18, 53-58]. Thus, questions remain as to whether digital 3D models or learning objects are more useful and effective in student learning than text or physical models.

Physical 3D anatomical models offer some advantages to students over simple illustrations, text, or even virtual models $[3,8]$. One mechanism that models may apply to learning is that of offloading cognition or the freeing of cognitive resources during learning [59]. Although this specific article examined learning in children, the hypothesis may have some significance in adults. As an example, a detailed physical model of the isolated sphenoid bone provides a student visual and tactile data concerning the size, regions, foramina, spines, and processes that are important in understanding the role of this bone in cranial organization. Thus, valuable cognitive resources may be redirected to understanding the correlation of cranial nerves and muscles to specific points on the model, rather than trying to understand the relationships between the greater and lesser wings of the sphenoid on a $2 \mathrm{D}$ illustration.

\section{Implementation of 3D Modeling and Printing in Medical and Dental Curricula}

\section{Digital 3D Anatomical Models}

Digital 3D anatomical models can applied in many ways in a medical or dental curriculum. Online materials, lecture presentations, instructor notes, and assessment can include 3D models of organs or tissues that are interactive (interactive PDFs) and enhanced in attention to specific structures. Characteristics of the models, such as resolution, color, scale, opacity, interactivity, time, and the inclusion of adjunct images or text, can be manipulated to enhance specific learning objectives or to target specific student populations. As described above, these models can be used as individual learning assets or as part of a larger learning object.

\section{Printed Anatomical Models}

The simplest implementation of printed 3D anatomical models into medical and dental curricula is as current anatomical models are used. The value of $3 \mathrm{D}$ printing lies in the choice of anatomical perspective, resolution, and scale. Instructors can now create models from specific perspectives, such as disarticulated skull bones, expanded models of the temporal bone, and pulmonary or vascular structures.

A limitation to the application of 3D printed models is the depiction of fascia. Anatomical education is incomplete without the student understanding fascia, fascial compartments, and connective tissue [60]. Physical anatomical models, either commercial, plastinized, or 3D printed, cannot depict fine fascial elements and connective tissue compartmentalization of organs and vessels. In curricula that do not engage in cadaver dissection or prosection, the students are at a disadvantage and must learn these concepts in postgraduate education.

Costs of printed 3D models have been cited as impediments to their implementation into the medical curriculum [61]. However, after the model is created and verified and with optimization of the printing parameters, subsequent prints are much less expensive than the first print or a quality commercial model. If costs go down with time, as is anticipated, it may be reasonable to print small models for all students to have and review themselves. There may come a time where ideal cadavers can be printed in their entirety in material suitable for "dissection," with incorporation of fascial elements, no constraints in variation or size, and in reasonable time.

\section{Clinical Case Studies}

Recently, Zein and colleagues reported that the 3D printing of the livers of living donors may improve preoperative surgical planning, reduce unnecessary surgeries, and decrease the complications of liver transplant surgery [62]. Similar preoperative organ studies have been performed by other groups [60, 63-67]. The 3D printed liver models, when linked to patient data, can be an effective learning object to teach anatomy, pathology, and radiology to medical students and residents. This learning object provides the student an opportunity to confirm imaging findings and to understand the relationship of the image with the 3D model or actual liver. While these concepts seem trivial, they are the basis of understanding the visual models created by CT or MRI and the application of those models to clinical cases. The difficult question is how to implement such an object into the medical curriculum.

Many medical schools have incorporated active learning methods in their medical curriculum, including small-group problem-based learning (PBL). In its usual form, PBL methods introduce basic science or clinical concepts to the medical student through specific clinical case presentations. This format fits well with the concept of using a comparison of 
digital imaging and 3D model construction to teach anatomy and radiology. This implementation can also be applied to other organ systems and clinical cases to include cardiopulmonary, renal, neural, and skeletal concepts. However, broadening of the implementation of this method would require a method of obtaining both usable 3D models and corresponding patient data. The collaboration of clinical radiologists to identify possible cases, 3D modelers to segment the patient data and create the physical 3D models, and medical school educators to design a complete learning object around these assets is required. In addition, there must be assurance that the patient data is handled according to Internal Review Board and Health Insurance Portability and Accountability Act of 1996 (HIPAA) guidelines. Further, the costs involved in the creation of printable models and the printing of these models in sufficient size, quantity, and resolution are substantial. These obstacles will likely be reduced with time by the creation of institutional and commercial case databases that can be accessed by association or license and the anticipated significant reduction in cost of $3 \mathrm{D}$ printers and materials.

Assessment of the impact of these new teaching methods on student learning is required to determine if they are worth the effort in full implementation. For schools with a fairly stable curriculum, simple comparisons of the student performance before and after implementation may be sufficient to consider continuing implementation. Other schools that may be transitioning between curricular structure and concepts would need to be more creative in their assessment protocol to separate outcomes from the influence of other significant curricular changes. In this case, extracurricular assessment may be required, such as random assignment of students to different learning groups that are exposed to different teaching methods or large focus group data. In either case, validation of the teaching methodology is critical to proceed with advances in implementation of these technologies in the medical curriculum.

\section{A Convergence of Digital 3D Modeling and 3D Printing}

The concept of "tangible multimedia" has been described as the linking of online educational content with a tangible object, which, together, represents the learning object [68]. Studies have examined the impact of this concept on learning in preschool children, whom they describe as having a "large learning gap" in the multimedia environment since they lack logical thinking and abstract reasoning. Medical and dental students do not lack logical thinking or abstract reasoning; however, they may have difficulty reconstructing a clear view of an anatomical structure or embryological event from a digital rendering, then applying that view to clinical concepts. A tangible object may enable the student to overcome this difficulty and enhance learning by reducing extraneous cognitive load. Thus, learning may be enhanced by linking multimedia educational material with a tangible object.

A direct application of tangible multimedia could involve the implementation of a digital simulation of human embryonic cardiovascular development in the medical curriculum. The material may consist of animations of primitive heart tube formation and heart looping, as well as atrial, ventricular, and outflow tract septation, all of which have been indicated by medical students in focus group data as conceptually difficult. Digital 3D models of normal hearts and hearts with congenital anomalies allow the user to view and manipulate renderings of specific anomalies and to confirm the information presented in the simulation. As the students complete the material and embedded assessment tools, they would be provided a 3D printed heart (tangible object) produced from the same digital 3D models observed in the animations and interactive materials. The models are printed in a form that provides repetition of the learning objectives and reinforcement of embryological concepts. The built-in assessment in the form of quizzes or possibly small games will allow tracking of student performance, as well as short-term goals to advance through the material. This format allows implementation of the material without disruption of the current curriculum. It can be treated as adjunct material or a principal teaching tool. What is unique here is that the students end the material with a tangible model that they can manipulate. This teaching tool can also be applied to patient and family education to strengthen informed consent and explore treatment choices.

\section{Future Considerations}

The prospects for application of digital 3D models and 3D printing in medical education are extensive. As the technologies advance, it is likely that current issues such as cost and safety will be addressed and become less significant.

A limitation in the application of 3D printing in medical education and a significant factor in cost is the availability of digital 3D models. A great idea for a printed model waits on the creation of a usable digital 3D model in a format recognized by a 3D printer. If the digital 3D model requires segmentation from clinical imaging or creation using 3D modeling software, it may take some time to complete. Time and effort spent by an engineer, technician, radiologist, or biomedical illustrator to segment or create a digital 3D model add to the costs. One solution is the creation of peer-reviewed digital 3D model libraries or repositories. Authors submit models that are reviewed and revised. If accepted, they are placed in the library for use in education. A small fee could be charged to support the operation of the library. While a basic set of models would be similar to a basic anatomy atlas, the number of models required to depict difficult anatomy, anatomical variations, and anomalies is vast. Searching a library and 
obtaining an appropriate model though licensing is much more cost effective than creating a model from scratch.

The implementation of 3D printing, as well as any new teaching tool into the curriculum, must be assessed for efficacy in learning. A positive assessment adds to the cost effectiveness of the material and the technology. Assessment in the case of digital learning objects can be imbedded in the material as quizzes, case studies, and even games. The embedded assessment along with tracking of user performance enables the entire learning object to be a stand-alone package that, when accessed, provides performance outcomes. Combine this type of assessment with evaluation of the "dissection" of a $3 \mathrm{D}$ printed organ or anatomical region and a complete record of student engagement in the material and learning outcome can be obtained, all without disruption of the current curriculum.

Safety in the process of 3D printing, the materials used, and assurances of good environmental stewardship are important to the advancement of $3 \mathrm{D}$ printing technologies. Information as to safe exposure rates to ultrafine particles and compounds emitted during 3D printing using specific materials should be provided to users, and users should be cognizant of the need to protect themselves and others from excessive exposure.

Acknowledgments The authors thank Dr. James Edmonson and Laura Travis of the Dittrick Museum at Case Western Reserve University for the ivory model image; Drs. Raymond Muzic, Joseph Molter, and Prabhakar Rajiah from University Hospitals for their help understanding medical imaging and segmentation; Kellie Pasini and staff at Thinkbox CWRU for their help with modeling and printing; and 3B Scientific for commercial heart model images. Some aspects of this study and review were funded by a Nord Grant through the University Center for Innovation in Teaching and Education at Case Western Reserve University.

Conflict of Interest The authors declare no conflict of interest.

Open Access This article is distributed under the terms of the Creative Commons Attribution License which permits any use, distribution, and reproduction in any medium, provided the original author(s) and the source are credited.

\section{References}

1. Marković D, Marković ŽB. Development of anatomical modelschronology. Acta Med Medianae. 2010;49:56-62.

2. Riva A, Conti G, Solinas P, Loy F. The evolution of anatomical illustration and wax modelling in Italy from the 16th to early 19th centuries. J Anat. 2010;216:209-22.

3. Pawlina W, Drake RL. Anatomical models: don't banish them from the anatomy laboratory yet. Anat Sci Educ. 2013;6:209-210.22.

4. Russell KF. Ivory anatomical manikins. Med Hist. 1972;16:13142.

5. Maerker A. Florentine anatomical models and the challenge of medical authority in late-eighteenth-century Vienna. Stud Hist Phil Biol Biomed Sci. 2012;43:730-40.

6. Hopwood N. The art of medicine. Lancet. 2008;372:1946-7.
7. Khot Z, Quinlan K, Norman GR, Wainman B. The relative effectiveness of computer-based and traditional resources for education in anatomy. Anat Sci Educ. 2013;6:211-5.

8. Preece D, Williams SB, Lam R, Weller R. "Let's get physical": advantages of a physical model over 3D computer models and textbooks in learning imaging anatomy. Anat Sci Educ. 2013;6: 216-24.

9. Lombardi SA, Hicks RE, Thompson KV, Marbach-Ad G. Are all hands-on activities equally effective? Effect of using plastic models, organ dissections, and virtual dissections on student learning and perceptions. Adv Physiol Educ. 2014;38:80-6.

10. Ruiz JG, Mintzer MJ, Issenberg SB. Learning objects in medical education. Med Teach. 2006;28:599-605.

11. Sugand K, Abrahams P, Khurana A. The anatomy of anatomy: a review for its modernization. Anat Sci Educ. 2010;3:83-93.

12. Ayres P, Paas F. Can the cognitive load approach make instructional animations more effective? Appl Cogn Psychol. 2007;21:811-20.

13. Mayer RE. Applying the science of learning to medical education. Med Educ. 2010;44:543-9.

14. Moreno R. Optimising learning from animations by minimising cognitive load: cognitive and affective consequences of signalling and segmentation methods. Appl Cogn Psychol. 2007;21:765-81.

15. Tabbers HK, Martens RL, van Merriënboer JJG. Multimedia instructions and cognitive load theory: effects of modality and cueing. Br J Educ Psychol. 2004;74:71-81.

16. Fredieu J, Watson J, Hughart C, Nikiforova T. Human development: development of the face and palate. MedEdPORTAL. 2011;8334.

17. $\mathrm{Hu} \mathrm{A}$, Wilson T, Ladak H, Haase P, Doyle P, Fung K. Evaluation of a three-dimensional educational computer model of the larynx: voicing a new direction. J Otolaryngol Head Neck Surg. 2010;39: 315-22.

18. Marsh KR, Giffin BF, Lowrie Jr DJ. Medical student retention of embryonic development: impact of the dimensions added by multimedia tutorials. Anat Sci Educ. 2008;1:252-7.

19. Tan S, Hu A, Wilson T, Ladak H, Haase P, Fung K. Role of a computer-generated three-dimensional laryngeal model in anatomy teaching for advanced learners. J Laryngol Otol. 2012;126:395401.

20. Yue C, Kim J, Ogawa R, Stark E, Kim S. Applying the cognitive theory of multimedia learning: an analysis of medical animations. Med Educ. 2013;47:375-87.

21. Garg AX, Norman GR, Eva KW, Spero L, Sharan S. Is there any real virtue of virtual reality?: the minor role of multiple orientations in learning anatomy from computers. Acad Med. 2002;77(10):S979.

22. Ruiz JG, Cook DA, Levinson AJ. Computer animations in medical education: a critical literature review. Med Educ. 2009;43:838-46.

23. Ballestriero R. Anatomical models and wax Venuses: art masterpieces or scientific craft works? J Anat. 2010;216:223-34.

24. Hopwood N (2002) Embryos in wax: models from the Ziegler Studio. Cambridge and Bern: Whipple Museum of the History of Science, University of Cambridge, and Institute of the History of Medicine, University of Bern.

25. Maerker A. Anatomizing the trade. Technol Cult. 2013;54:531-62.

26. Guyer RL. Metamorphosis: beautiful education to smarmy edutainment. Am J Bioeth. 2007;7:30.

27. Valdecasas A, Correas A, Guerrero C, Juez J. Understanding complex systems: lessons from Auzoux's and von Hagens's anatomical models. J Biosci. 2009;34:835-43.

28. Von Hagens G. Impregnation of soft biological specimens with thermosetting resins and elastomers. Anat Rec. 1979;194:247-55.

29. Douglass C, Glover R. Plastination: preservation technology enhances biology teaching. Am Biol Teach (Natl Assoc Biol Teach). 2003;65:503. 
30. Latorre RM, García-Sanz MP, Moreno M, Hernández F, Gil F, López $\mathrm{O}$, et al. How useful is plastination in learning anatomy? Vet Med Educ. 2007;34:172-6.

31. Jones DG. Re-inventing anatomy: the impact of plastination on how we see the human body. Clin Anat. 2002;15:436-40.

32. Becker BW. Digital learning object repositories. Behav Soc Sci Libr. 2010;29:86-8.

33. Kerfoot E, Lamata P, Niederer S, Hose R, Spaan J, Smith N. Share and enjoy: anatomical models database - generating and sharing cardiovascular model data using web services. Med Biol Eng Comput. 2013;51:1181-90.

34. Richards G, McGreal R, Hatala M, Friesen N. The evolution of learning object repository technologies: portals for on-line objects for learning. J Dist Educ. 2002;17:67-79.

35. Wulf J, Rohde I, Koppe T, Winder RJ. Three-dimensional microimaging $(\mu \mathrm{CT})$ based physical anatomic teaching models: implementation of a new learning aid for routine use in anatomy lectures. Stud Health Technol Inform. 2012;173:549-51.

36. McMenamin PG, Quayle MR, McHenry CR, Adams JW. The production of anatomical teaching resources using three-dimensional (3D) printing technology. Anat Sci Educ. 2014. doi:10.1002/ase. 1475. Article first published online.

37. Heimann T, Meinzer H. Statistical shape models for 3D medical image segmentation: a review. Med Image Anal. 2009;13:543-63.

38. van Rikxoort EM, van Ginneken B. Automated segmentation of pulmonary structures in thoracic computed tomography scans: a review. Phys Med Biol. 2013;58:R187-220.

39. Zhuang X. Challenges and methodologies of fully automatic whole heart segmentation: a review. J Healthc Eng. 2013;4:371-408.

40. Huang S, Liu P, Mokasdar A, Hou L. Additive manufacturing and its societal impact: a literature review. Int J Adv Manuf Technol. 2013;67:1191-203.

41. Hull CW. Apparatus for production of three-dimensional objects by stereolithography. Patent. 1986; 4575330, Mar 11.

42. Deckard C, Beaman J. Recent advances in selective laser sintering, 14th Conference on Production Research in Technology (University of Michigan). 1987; pp. 447-451.

43. Crump S. Fused deposition modeling (FDM): putting rapid back in prototyping, 2nd Int. Conf. on Rapid Prototyping. 1991; p. 358-361

44. Salmi M, Paloheimo K, Tuomi J, Wolff J, Mäkitie A. Accuracy of medical models made by additive manufacturing (rapid manufacturing). J Craniomaxillofac Surg. 2013;41:603-9.

45. Stephens B, Azimi P, El Orch Z, Ramos T. Ultrafine particle emissions from desktop 3D printers. Atmos Environ. 2013;79:334-9.

46. Andersen ZJ, Olsen TS, Andersen KK, Loft S, Ketzel M, Raaschou-Nielsen O. Association between short-term exposure to ultrafine particles and hospital admissions for stroke in Copenhagen, Denmark. Eur Heart J. 2010;31:2034-40.

47. Peters A, Wichmann HE, Tuch T, Heinrich J, Heyder J. Respiratory effects are associated with the number of ultrafine particles. Am J Respir Crit Care Med. 1997;155:1376-83.

48. Schmid O, Möller W, Semmler-Behnke M, Ferron GA, Karg E, Lipka J, et al. Dosimetry and toxicology of inhaled ultrafine particles. Biomarkers. 2009;14 Suppl 1:67-73.

49. Shannahan JH, Kodavanti UP, Brown JM. Manufactured and airborne nanoparticle cardiopulmonary interactions: a review of mechanisms and the possible contribution of mast cells. Inhal Toxicol. 2012;24:320-39.

50. Schaper MM, Thompson RD, Detwiler-Okabayashi K. Respiratory responses of mice exposed to thermal decomposition products from polymers heated at and above workplace processing temperatures. Am Ind Hyg Assoc J. 1994;55:924-34.

51. Shapi MM, Hesso A. Gas chromatographic-mass spectrometric analysis of some potential toxicants amongst volatile compounds emitted during large-scale thermal degradation of poly (acrylonitrile-butadiene-styrene) plastic. J Chromatogr. 1991;562:681-96.

52. Falloon G, Janson R, Janson A. Digital learning objects: a need for educational leadership. Ágora. 2009;44:48-53.

53. Farha NW. An exploratory study into the efficacy of learning objects. J Educ Online. 2009;6:1-32.

54. Jenkinson J. Measuring the effectiveness of educational technology. Proc Int Conf e-Learn. 2009;222-227.

55. Keedy AW, Durack JC, Sandhu P, Chen EM, O'Sullivan PS, Breiman RS. Comparison of traditional methods with 3D computer models in the instruction of hepatobiliary anatomy. Anat Sci Educ. 2011:4:84-91.

56. McNulty JA, Sonntag B, Sinacore JM. Evaluation of computeraided instruction in a gross anatomy course: a six-year study. Anat Sci Educ. 2009;2:2-8.

57. Nguyen N, Nelson AJ, Wilson TD. Computer visualizations: factors that influence spatial anatomy comprehension. Anat Sci Educ. 2012;5:98-108.

58. Sergovich A, Johnson M, Wilson TD. Explorable threedimensional digital model of the female pelvis, pelvic contents, and perineum for anatomical education. Anat Sci Educ. 2010;3: $127-33$.

59. Manches A, O'Malley C. Tangibles for learning: a representational analysis of physical manipulation. Pers Ubiquit Comput. 2012;16: 405-19.

60. Korf H, Wicht H, Snipes RL, Timmermans J, Paulsen F, Rune G, et al. The dissection course - necessary and indispensable for teaching anatomy to medical students. Ann Anat. 2008;190:16-22.

61. Rengier F, Mehndiratta A, von Tengg-Kobligk H, Zechmann CM, Unterhinninghofen R, Kauczor H, et al. 3D printing based on imaging data: review of medical applications. Int J Comput Assist Radiol Surg. 2010;5:335-41.

62. Zein NN, Hanouneh IA, Bishop PD, Samaan M, Eghtesad B, Quintini C, et al. Three-dimensional print of a liver for preoperative planning in living donor liver transplantation. Liver Transpl. 2013;19:1304-10.

63. Beermann J, Tetzlaff R, Bruckner T, Schöebinger M, Müller-Stich $\mathrm{BP}$, Gutt CN, et al. Three-dimensional visualisation improves understanding of surgical liver anatomy. Med Educ. 2010;44:936-40.

64. Cohen A, Laviv A, Berman P, Nashef R, Abu-Tair J. Mandibular reconstruction using stereolithographic 3-dimensional printing modeling technology. Oral Surg Oral Med Oral Pathol Oral Radiol Endod. 2009;108:661-6.

65. Ikegami T, Maehara Y. Transplantation: 3D printing of the liver in living donor liver transplantation. Nat Rev Gastroenterol Hepatol. 2013;10:697-8.

66. Thomas D, Azmi M, Tehrani Z. 3D additive manufacture of oral and maxillofacial surgical models for preoperative planning. Int $\mathrm{J}$ Adv Manuf Technol. 2014;71:1643-51.

67. Xiao K, Zardawi F, Noort R, Yates J. Developing a 3D colour image reproduction system for additive manufacturing of facial prostheses. Int J Adv Manuf Technol. 2014;70:2043-9.

68. Tsong CK, Toh SC, Samsudin Z. Tangible multimedia: a case study for bringing tangibility into multimedia learning. Turkish Online J Educ Technol. 2012;11:442-50. 